\title{
Ultrassonografia esplênica, hepática, biliar e renal em caprinos: uma revisão
}

\author{
[Splenic, hepatic, biliary, and renal ultrasonography in goats. A review]
}

\section{"Revisão/Review"}

\author{
Francisco Carlos de Sousa ${ }^{1}$, Dárcio Ítalo Alves Teixeira ${ }^{2 *}$
}

\author{
${ }^{1}$ Instituto Federal de Educação, Ciência e Tecnologia do Ceará, Umirim-CE, Brasil. \\ ${ }^{2}$ Universidade Estadual do Ceará, Fortaleza-CE, Brasil. \\ *Autor para correspondência/Corresponding author: E-mail: diat@uece.br
}

\begin{abstract}
Resumo
A utilização da ultrassonografia bidimensional é crescente na clínica de ruminantes, principalmente na avaliação abdominal destes animais. Esta é uma ferramenta segura, prática, rápida, não invasiva e sem efeito colateral conhecido tanto para o paciente quanto para o examinador. A ultrassonografia permite a avaliação de órgãos como fígado, baço, rins, abomaso, rúmen, retículo, órgãos reprodutivos e outros, fornecendo informação a respeito da localização anatômica, tamanho e características da ecotextura do parênquima, deteç̧ão de anormalidades focais ou difusamente distribuídas no parênquima, por exemplo. Estudos relacionados ao uso da ultrassonografia podem ser de grande valia no exame desses órgãos, podendo prover aos clínicos informações diagnósticas específicas, anteriormente disponíveis somente por laparotomia exploratória. Assim, este estudo teve por objetivo fornecer informações referentes à utilização da ultrassonografia para descrição da anatomia e diagnóstico de alterações morfofuncionais em órgãos como baço, fígado, vesícula biliar e rins de caprinos sadios.
\end{abstract}

Palavras-chave: exame clínico; baço; fígado; rins; ultrassom.

\begin{abstract}
The use of two-dimensional ultrasonography is increasing in the ruminant clinic, mainly for the abdominal evaluation of these animals. It is safe, practical, quick, non-invasive, and without known collateral effects for both the patient and the examiner. Ultrasound imaging allows the evaluation of organs such as liver, spleen, kidneys, abomasum, rumen, reticulum, reproductive organs, and others, providing information about to the anatomical location, size, and characteristics of the parenchyma echotexture, detection of focal or diffusely distributed abnormalities. Studies about the use of ultrasonography can be very useful in the examination of abdominal organs and can provide information previously available only by exploratory laparotomy. Thus, the aim of this review is to provide information regarding the use of ultrasound technique for anatomy description and diagnosis of morphological and functional alterations in organs such as the spleen, liver, gallbladder, and kidneys of healthy goats.
\end{abstract}

Keywords: clinical examination; spleen; liver; kidneys; ultrasound.

\section{Introdução}

O rebanho caprino brasileiro é estimado em 9,6 milhões de animais, sendo a região Nordeste detentora da maior parte desse efetivo $(8,9$ milhões), principalmente os estados da Bahia, Pernambuco, Piauí e Ceará. A caprinocultura tem se destacado no agronegócio brasileiro; carne, leite e pele estão entre os principais produtos comercializados (IBGE, 2017). Diante deste cenário produtivo, torna-se necessária a utilização de métodos que auxiliem o clínico durante a avaliação abdominal, tornando assim mais assertivos os diagnósticos dos distúrbios esplênicos, hepáticos e renais, os quais podem afetar diretamente a produtividade dos animais e que por vezes podem levar o animal a óbito.

O diagnóstico de distúrbios esplênicos, hepáticos e renais em ruminantes pode ser 
realizado com base em sinais clínicos, exame histopatológico de biópsias, análise hematológica e bioquímica, porém esses apresentam certas limitações. Não há sinais clínicos característicos de alteração esplênica, nem exames laboratoriais específicos para o exame deste órgão em caprinos. Provas bioquímicas representam um excelente subsídio ao diagnóstico clínico de alterações hepáticas e renais, mas podem não refletir a magnitude da doença e não ser específicos para uma determinada enfermidade. $\mathrm{O}$ procedimento de biópsia associado com o diagnóstico histopatológico, embora seja o método mais acurado, não pode ser utilizado rotineiramente, uma vez que é um teste laborioso, invasivo e requer o uso de anestesia (Acorda et al., 2009).

A ultrassonografia bidimensional, por sua vez, é um método importante de diagnóstico, por permitir a avaliação e o estudo dos órgãos internos de forma contínua e em tempo real, não invasiva e segura, tanto para o paciente, quanto para o operador (King, 2006). O primeiro relato do uso do ultrassom como método diagnóstico na Medicina Veterinária foi a identificação de gestação em ovinos (Lindahl, 1966), realizada no serviço de pesquisa de agricultura e pecuária em
Belsville, Estados Unidos da América. A introdução da ultrassonografia como meio diagnóstico possibilitou a obtenção de informações precisas, referentes a tamanho, forma, arquitetura interna, ecotextura e contorno dos órgãos em estudo (Simões, 2008).

A ultrassonografia desempenha um papel proeminente na história da Medicina veterinária. Ganhando popularidade na clínica de pequenos animais em meados dos anos 80, esta ferramenta vem se mostrando um importante procedimento investigativo, auxiliando no diagnóstico de lesões, pois permite avaliar e caracterizar a aparência normal e alterada dos diferentes órgãos da cavidade abdominal (Nyland e Matton, 1995).

Apesar disso, esta ferramenta ainda é subutilizada em caprinos, sendo aplicada principalmente para o exame de órgãos reprodutivos. Assim, o uso do ultrassom para o exame clínico abdominal nessa espécie é relativamente recente, com a primeira publicação datada de 1996. A Tabela 1 traz uma compilação de trabalhos que empregaram a ultrassonografia para o exame de baço, fígado, vesícula biliar e rins em caprinos, utilizando diferentes transdutores.

Tabela 1. Tipo de transdutores e objetivos do uso da ultrassonografia no exame de baço, fígado, vesícula biliar e rins em caprinos.

\begin{tabular}{|c|c|c|c|}
\hline Órgão & $\begin{array}{c}\text { Transdutor/Frequência } \\
\text { (MHz) }\end{array}$ & Finalidade & Referência \\
\hline Baço & $\begin{array}{c}\text { Convexo/3,5 } \\
\text { Linear } / 5,0\end{array}$ & $\begin{array}{l}\text { Descritiva } \\
\text { Descritiva }\end{array}$ & $\begin{array}{c}\text { Acorda et al., } 2009 \\
\text { Braun e Steininger, } 2010\end{array}$ \\
\hline \multirow[t]{2}{*}{$\begin{array}{l}\text { Fígado/ } \\
\text { Vesícula } \\
\text { biliar }\end{array}$} & $\begin{array}{c}\text { Linear } / 5,0 \\
\text { Convexo/3,5 } \\
\text { Linear/3,5 }\end{array}$ & $\begin{array}{c}\text { Descritiva } \\
\text { Descritiva } \\
\text { Diagnóstico de } \\
\text { cisto hidático }\end{array}$ & $\begin{array}{c}\text { Braun e Steininger, } 2011 \\
\text { Acorda et al., } 2006 \\
\text { Maxson et al., } 1996\end{array}$ \\
\hline & Setorial/3,5 & Descritiva & Kandeel et al., 2009 \\
\hline \multirow[t]{2}{*}{ Rins } & $\begin{array}{l}\text { Convexo/3,5 } \\
\text { Linear/6,0 }\end{array}$ & $\begin{array}{l}\text { Descritiva } \\
\text { Diagnóstico de } \\
\text { hidronefrose }\end{array}$ & $\begin{array}{c}\text { Acorda et al., } 2009 \\
\text { El-Kammar et al., } 2012\end{array}$ \\
\hline & Não informado & $\begin{array}{l}\text { Diagnóstico de } \\
\text { hidronefrose }\end{array}$ & Newman et al., 2000 \\
\hline
\end{tabular}

Em caprinos, a ultrassonografia tem sido empregada principalmente para o estudo de eventos reprodutivos de fêmeas, tais como avaliação de estruturas ovarianas (Teixeira et al., 2008; Sousa et al., 2011), diagnóstico de gestação, determinação da viabilidade, número, idade e sexo fetal (Iswar, 1995; Teixeira et al., 2009; Medan e
El-Aty, 2010). Também tem sido utilizada na avaliação do úbere, sendo empregada para a descrição da estrutura interna da glândula mamária durante a lactação (Fasulkov et al., 2010), bem como para a estimativa da produção leiteira desses animais (Ayadi et al., 2003; Salama et al., 2004; Melo et al., 2012). Em caprinos machos, a 
aparência ultrassonográfica de testículos e epidídimos foi descrita por Ahmad et al. (1991), evidenciando sua importante aplicação no exame andrológico. Recentemente, no entanto, nosso grupo mostrou a importância do exame ultrassonográfico em outros sistemas ao concluir que a ultrassonografia foi tão eficiente quanto a laparoscopia, quando utilizada como ferramenta auxiliar na obtenção de biópsias de baço e fígado em caprinos machos transgênicos (Melo et al., 2015).

Dessa forma, estudos relacionados ao uso da ultrassonografia são de grande valia no exame desses órgãos, podendo prover aos clínicos informações diagnósticas específicas, anteriormente disponíveis somente por laparotomia exploratória, possibilitando avaliação de forma não invasiva e rápida. Assim, no presente artigo de revisão, tópicos sobre a utilização da ultrassonografia para descrição da anatomia e diagnóstico de alterações morfofuncionais em órgãos como baço, fígado, vesícula biliar e rins de caprinos sadios foram revisados.

\section{Ultrassonografia esplênica}

Em caprinos, o baço está localizado dorsalmente do $10^{\circ}$ ao $12^{\circ}$ espaço intercostal (EIC) no quadrante abdominal esquerdo. $\mathrm{O}$ baço é quadrilateral em caprinos, está situado sobre a parte craniodorsal do rúmen, contra a metade esquerda do diafragma, e é fixado aos dois órgãos (Dyce et al., 2010). Ele é envolto por uma cápsula de tecido mole, rica em fibras musculares e que projeta trabéculas no órgão. O baço é o maior órgão linfoide e várias funções lhe são atribuídas: ele armazena e concentra eritrócitos e os libera quando necessário; filtra o sangue e remove eritrócitos desgastados da circulação; extrai ferro da hemoglobina e o libera novamente para reutilização; produz linfócitos e monócitos e desempenha uma função importante na produção de anticorpos específicos em resposta a doenças sistêmicas; o baço está moderadamente aumentado em muitas doenças infecciosas, refletindo aumento da sua função (Königh e Liebich, 2011).

Previamente ao exame ultrassonográfico do baço em caprinos, deve ser realizada tricotomia da parede torácica esquerda, no espaço compreendido entre a $5^{\text {a }}$ costela e a fossa paralombar, da linha média dorsal à linha alba, permitindo a visualização das margens dorsal e ventral do órgão (Acorda et al., 2009). Na avaliação de estruturas esplênicas em caprinos, transdutores convexos com frequência de 3,5 $\mathrm{MHz}$ (Acorda et al., 2009) e lineares de 5,0 MHz (Braun e Steininger, 2010) têm sido empregados. $\mathrm{O}$ baço pode ser facilmente localizado do $8^{\circ}$ EIC até a região imediatamente caudal à última costela (Braun e Steininger, 2010). Segundo esses autores, a melhor área para visualização do parênquima esplênico está situada no aspecto dorsal do flanco esquerdo, do $10^{\circ}$ ao $12^{\circ}$ EIC. Em nossa experiência com caprinos da raça Canindé, a área anatômica que permite melhor visualização do parênquima esplênico está situada no aspecto dorsal do flanco esquerdo, do $10^{\circ}$ ao $12^{\circ}$ EIC, podendo se estender caudalmente à $13^{\mathrm{a}}$ costela em alguns animais, de forma não patológica (Sousa et al., 2016).

As estruturas do baço caprino que podem ser visualizadas por ultrassonografia são cápsula, parênquima e vasos. A cápsula do baço é hiperecoica e aparece delimitando a borda distal do órgão, permitindo sua fácil visualização (Acorda et al., 2009). O parênquima esplênico consiste de ecos fracos distribuídos homogeneamente por toda a extensão visível do órgão (Figura 1). A aparência ultrassonográfica do parênquima esplênico caprino é similar à descrita para pequenos animais (Nyland e Matoon, 1995) e bovinos (Imran et al., 2011). Apesar disso, em caprinos a ecogenicidade deste órgão pode ser um pouco variável. Em cabras Saanen, o baço mostrou-se hiperecoico em relação ao fígado em 17 dos 30 animais avaliados por ultrassonografia. Os vasos esplênicos são visualizados internamente no parênquima do órgão, aparecendo como estruturas anecoicas de formato circular a oval ou alongado. Em caprinos, o hilo esplênico não é visível por ultrassonografia, pois se encontra encoberto pelo pulmão (Braun e Steininger, 2010).

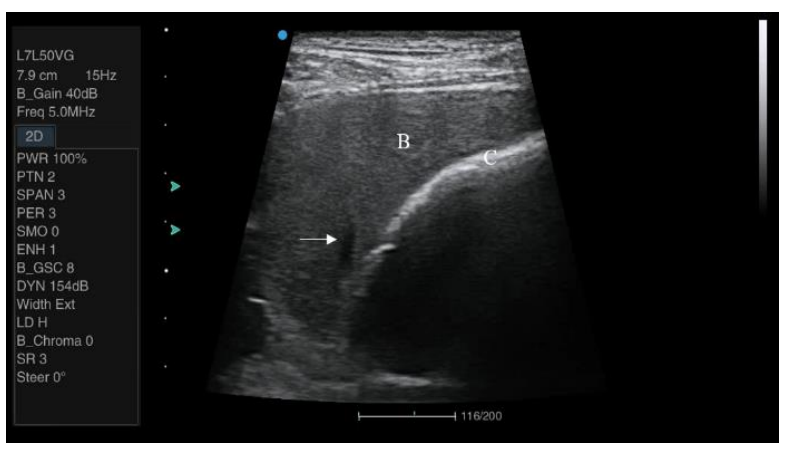

Figura 1. Imagem ultrassonográfica de exame transabdominal do baço de caprino Canindé de quatro anos de idade. A imagem foi obtida utilizando transdutor linear com frequência de 5,0 MHz, que foi posicionado no $11^{\circ}$ espaço intercostal do flanco esquerdo, em plano longitudinal. Baço: B; cápsula esplênica: C; veia tubular esplênica: seta. 
Em um estudo realizado por Acorda et al. (2009), no qual o exame ultrassonográfico do baço em caprinos e ovinos vivos foi comparado com a avaliação macroscópica post mortem, não foram observadas diferenças quanto à localização e às dimensões esplênicas. Desta forma, a avaliação do baço por ultrassonografia é útil clinicamente para determinar seu tamanho, localização e a presença de anormalidades no órgão, sempre que se suspeita de uma condição patológica (Acorda et al., 2009). Lesões focais ou difusamente distribuídas no baço podem ser vistas como mudanças no padrão de ecotextura do mesmo. O baço da espécie caprina pode ser afetado por tumores ou abscessos, no entanto, ao contrário dos bovinos, estes últimos são muito incomuns em caprinos, pois a reticuloperitonite traumática é rara (Braun et al., 2013).

\section{Ultrassonografia hepática e vesícula biliar}

$O$ fígado do ruminante adulto se aloja quase totalmente na metade direita do abdome, relacionado com a face caudal do diafragma e sob cobertura das costelas. Sua projeção se estende entre o terço ventral do $6^{\circ}$ EIC até a parte dorsal do último. O fígado é mantido em posição por certos ligamentos que o fixam ao diafragma e, mais importante, pela pressão visceral. A face livre do fígado é revestida quase totalmente pelo peritônio, o qual forma sua serosa. Ele se funde à cápsula fibrosa subjacente, que envolve todo o órgão (Dyce et al., 2010; Königh e Liebich, 2011).

O fígado é a maior glândula do corpo e tem função exócrina e endócrina. Seu produto exócrino, a bile, é armazenado e concentrado na vesícula biliar (VB) antes de escoar para o duodeno. A bile é responsável por emulsificar os componentes graxos antes da absorção. Ela também contém os produtos finais do metabolismo da hemoglobina e os subprodutos de determinados fármacos metabolizados. Substâncias endócrinas hepáticas são liberadas na corrente sanguínea e contribuem para o metabolismo de gorduras, carboidratos e proteínas; ele funciona como um depósito de glicogênio e, em animais jovens, funciona como um órgão hematopoiético (Königh e Liebich, 2011).

O exame ultrassonográfico hepático em caprinos é realizado após tricotomia da parede torácica direita, no espaço compreendido entre o $5^{\circ}$ e o $12^{\circ}$ EIC, da linha média dorsal à linha média ventral, permitindo a visualização das margens dorsal e ventral do fígado (Braun et al., 2013). Os EIC são examinados nos sentidos craniocaudal e dorsoventral, desde o $8^{\circ}$ até o $12^{\circ}$ EIC, com aparelho de ultrassonografia equipado com transdutor linear de 3,5 ou 5,0 MHz. Nessas varreduras, podem ser observados o aspecto ultrassonográfico do parênquima hepático, a localização da veia cava caudal (VCC), veia hepática $(\mathrm{VH})$, veia porta (VP) e VB, dependendo do EIC examinado. No $8^{\circ}$ EIC, o acesso ultrassonográfico se restringe à margem ventral do órgão, com maior alcance na fase expiratória da respiração. A partir do $9^{\circ}$ EIC, entretanto, a parte dorsal do parênquima hepático e a VCC situam-se sob o pulmão direito e só podem ser examinadas nos animais de menor porte, também durante a expiração. Do $10^{\circ}$ ao $12^{\circ}$ EIC há pouca interferência do pulmão direito sobre a formação da imagem hepática (Soroori et al., 2008).

O padrão ultrassonográfico do parênquima hepático (Figura 2) consiste de numerosos ecos fracos distribuídos de forma homogênea por toda a área do órgão e apresenta, nitidamente, maior ecogenicidade que o córtex renal direito (Kandeel et al., 2009). Um padrão de ecotextura hepática semelhante tem sido relatado em ovinos (Néspoli et al., 2009) e bovinos (Imran et al., 2011). Em caprinos, a superfície diafragmática do fígado aparece como uma linha ecoica suave e estreita, posicionada imediatamente adjacente ao peritônio e que se move em sincronia com o diafragma durante a respiração (Braun e Steininger, 2011). A superfície visceral do fígado também aparece como uma linha ecogênica estreita, mas não claramente definida do $5^{\circ}$ ao $10^{\circ}$ EIC, onde o fígado é delimitado pelo retículo e omaso, ou do $11^{\circ}$ ao $12^{\circ}$ EIC, devido à presença de alças intestinais que sobrepõem parcialmente $o$ parênquima hepático (Braun e Steininger, 2011). A vascularização hepática é representada por estruturas de lúmen anecoico, no entanto, enquanto a VH consiste de paredes hipoecoicas, a VCC e a VP apresentam limites bem definidos e paredes hiperecoicas (Acorda et al., 2006). Os segmentos longitudinais e transversais destes vasos podem ser identificados por ultrassonografia (Kandeel et al., 2009).

Normalmente, em secções transversais a VCC tem um formato oval a triangular, mas a VP sempre apresenta forma circular (Soroori et al., 2008). A VB aparece como uma estrutura preenchida por líquido, de formatos variáveis oval, oval a redondo ou redondo, com lúmen 
anecoico e paredes ecoicas. Seu diâmetro varia grandemente, dependendo do grau de repleção da mesma. Diferenças no diâmetro da VB não são observadas entre machos e fêmeas caprinas. Quando não apresentam alterações em sua estrutura, os ductos biliares não são visíveis no parênquima do fígado (Acorda et al., 2006; Kandeel et al., 2009).

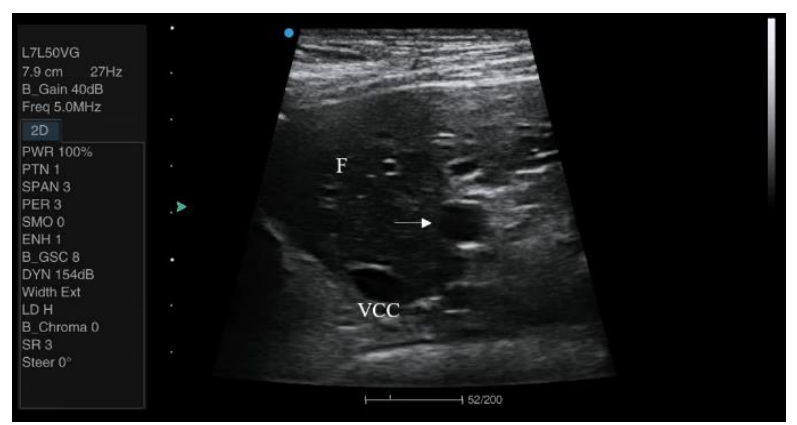

Figura 2. Imagem ultrassonográfica de exame transabdominal do fígado de caprino Canindé de quatro anos de idade. A imagem foi obtida utilizando transdutor linear com frequência de 5,0 $\mathrm{MHz}$, que foi posicionado no $8^{\circ}$ espaço intercostal do flanco direito, em plano longitudinal. Fígado: F; veia cava caudal: VCC; veia porta: seta.

Nos animais domésticos, as anormalidades focais do parênquima hepático e de estruturas relacionadas são produzidas por obstrução, colélitos, cistos, hemorragia, hematomas, abscessos, necrose, hiperplasia nodular e granuloma (Acorda, 1997). A ultrassonografia auxilia no diagnóstico dessas alterações hepáticas, pois, de modo geral, elas contrastam bem com o parênquima uniforme do fígado.

\section{Ultrassonografia renal}

Os rins são estruturas pares que se situam retroperitonealmente comprimidos contra a parede abdominal dorsal. Eles se localizam predominantemente na região lombar, mas se projetam cranialmente sob as últimas costelas para a parte intratorácica do abdome. $\mathrm{O}$ rim direito se situa mais cranialmente que o rim esquerdo e sua extremidade cranial faz contato com o fígado. Ele se posiciona em uma fossa do fígado (impressio renalis), a qual ajuda a limitar sua movimentação. O rim esquerdo possui maior mobilidade, já que não há impressão equivalente no fígado. Nos ruminantes, o tamanho considerável do rúmen empurra o rim esquerdo em direção à metade direita do abdome, onde ele é suspenso pelo longo e móvel mesonefro, caudal ao rim direito. Ambos os rins são envoltos em tecido adiposo, que os protege contra a pressão dos órgãos vizinhos. A forma básica dos rins de pequenos ruminantes se assemelha a um feijão. O parênquima renal é envolto por uma cápsula fibrosa resistente, que adentra no aspecto medial do rim para revestir as paredes do seio renal (Königh e Liebich, 2011).

A função principal do rim é manter a composição dos líquidos corporais dentro do âmbito fisiológico. Ele remove produtos finais do metabolismo e excreta substâncias do sangue pela filtração do plasma, inicialmente obtendo um grande volume de líquidos, o ultrafiltrado, também chamado de urina primária. O ultrafiltrado é sujeito a um novo processamento mediante o qual substâncias úteis (p. ex., água, glicose, eletrólitos e aminoácidos) são reabsorvidas de forma seletiva e substâncias desnecessárias são concentradas para eliminação. $\mathrm{O}$ produto final desses processamentos é a urina secundária. Também possuem funções endócrinas, sendo responsáveis pela produção dos seguintes hormônios: renina, bradicinina e eritropoietina (Königh e Liebich, 2011).

A avaliação ultrassonográfica dos rins em caprinos é realizada após tricotomia da fossa paralombar direita, no espaço compreendido entre o $10^{\circ}$ EIC e a última vértebra lombar, estendendose da linha média dorsal à linha média ventral. A visualização do rim direito é conseguida posicionando-se o transdutor na região dorsal do flanco direito, na fossa paralombar (Acorda et al., 2005). Em nossa experiência, a área que permitiu obter melhor visualização desse órgão foi a região imediatamente caudal à última costela, perto da linha mediana da parede abdominal (Sousa et al., 2016). Desta forma, o rim direito caprino é facilmente observado por ultrassonografia, contrariamente ao que tem sido relatado para o rim esquerdo. Em um estudo realizado por Acorda et al. (2005), após inúmeras tentativas de se visualizar o rim esquerdo, seja posicionando o transdutor na fossa paralombar esquerda ou direita, o mesmo não foi observado. Segundo esses autores, o rim esquerdo caprino é sobreposto pelo saco craniodorsal do rúmen, à esquerda, e pelo cólon transverso e descendente, no flanco direito, tornando difícil sua visualização. Apesar disso, em um trabalho realizado por Braun et al. (1992) foi possível visualizar ambos os rins em todos os 62 animais da espécie ovina examinados. Nesse estudo, o rim esquerdo foi localizado na fossa paralombar direita, caudalmente ao rim direito.

Várias estruturas que compõem o rim podem ser distinguidas durante o exame ultrassonográfico 
(Yamaga e Too, 1984). O córtex renal tem padrão hiperecoico e a pelve renal, estrutura centralmente localizada, também aparece ecoica. A medula renal é hipoecoica e está localizada entre o córtex hiperecoico e a pelve; as pirâmides medulares podem ser identificadas como estruturas circulares hipoecoicas (Figura 3). Comparando machos e fêmeas caprinas nativas das Filipinas, não foram observadas diferenças na aparência ultrassonográfica normal das diferentes estruturas renais (Acorda et al., 2005). Em equinos e caninos, o rim aparece com o mesmo padrão ultrassonográfico de caprinos, similar ao descrito acima para córtex, medula, pelve e pirâmides renais (Yamaga e Too, 1984).

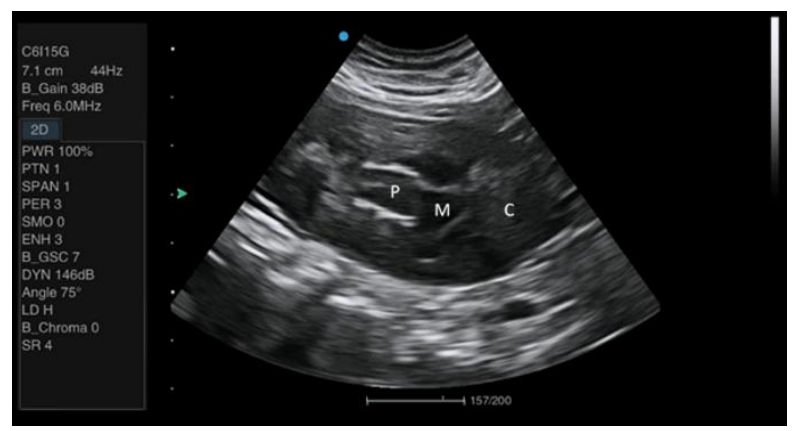

Figura 3. Imagem ultrassonográfica de exame transabdominal do rim direito de caprino Canindé de quatro anos de idade. A imagem foi obtida utilizando transdutor convexo com frequência de $6,0 \mathrm{MHz}$, o qual foi posicionado caudalmente à $13^{\mathrm{a}}$ costela, em plano longitudinal lateral. Córtex: C; medula: M; pelve: P.

Por meio da avaliação ultrassonográfica, informações importantes relacionadas ao tamanho, ao formato e à arquitetura interna renais podem ser obtidas. Segundo Streeter e Step (2007), a ultrassonografia renal é útil para diagnosticar pielonefrite e urolitíase. De acordo com esses autores, mudanças ultrassonográficas associadas à pielonefrite incluem dilatação renal, dilatação e acúmulo de material ecoico dentro do sistema coletor, bem como dilatação ureteral. Se a arquitetura renal é alterada por pielonefrite o prognóstico é desfavorável. Outras enfermidades que podem alterar a arquitetura renal, tais como cistos, abscessos e tumores renais também podem ser identificadas por ultrassonografia transretal ou transabdominal (Kofler e Hittmair, 2006). Em um trabalho realizado por Newman et al. (2000), a ultrassonografia abdominal em um caprino Pigmeu confirmou dilatação renal e hidronefrose bilateral. Após procedimento cirúrgico experimental de ligadura ureteral em caprinos Baladi, o exame ultrassonográfico permitiu diagnosticar hidronefrose, com base no aumento significativo de comprimento e largura do rim, bem como dilatação da pelve renal (El-Kammar et al., 2012).

\section{Considerações Finais}

A ultrassonografia é uma importante ferramenta de diagnóstico por imagem que permite avaliar, in vivo e de forma não invasiva, parâmetros como localização, tamanho, forma, arquitetura interna e ecotextura de órgãos em funcionamento, ou o monitoramento de suas funções. Particularmente na espécie caprina, a ultrassonografia pode ser empregada com a mesma praticidade que em pequenos animais, permitindo realizar o exame de baço, fígado, vesícula biliar e rins.

As informações apresentadas neste artigo revelam a importância da utilização da ultrassonografia como ferramenta para o clínico de animais de produção. Inicialmente aplicando-a para explorar as imagens dos órgãos da cavidade abdominal, como baço, fígado e rins, assim como visibilizar lesões morfofuncionais, guiando o buiatra a diagnósticos assertivos na clínica de caprinos.

\section{Referências}

Acorda, J.A. Ultrasonographic diagnosis of hepatic diseases in animals. Philippine Journal of Veterinary Medicine, 34(2): 68-75, 1997.

Acorda, J.A.; Ancheta, M.N.; Detera, M.A.J.; Cabrera, L.A.; Maligaya, R.L. Comparative ultrasound features and echo histograms of the spleen in female goats (Capra hircus), sheep (Ovis aries) and buffaloes (Bubalus bubalis). Philippine Journal of Veterinary and Animal Sciences, 35(2): 135-146, 2009.

Acorda, J.A.; Acebedo, M.C.; Maligaya, L. Ultrasonographic features of the liver and associated structures in Philippine native goats (Capra hircus). Philippine Journal of Veterinary Medicine, 43(1): 1-7, 2006.

Acorda, J.A.; Vergara, M.E.M.; Maligaya, R.L. Ultrasonographic examination of the kidneys in Philippine native goats (Capra hircus). Philippine Journal of Veterinary Medicine, 42(1): 9-15, 2005.

Ahmad, N.; Noakes, D.E.; Subandrio, A.L. Bmode real time ultrasonographic imaging of tile testis and epididymis of sheep and goats. Veterinary Record, 128(21): 491-496, 1991. 
Ayadi, M.; Caja, G.; Such, X.; Knight, C.H. Use of ultrasonography to estimate cistern size and milk storage at different milking intervals in the udder of dairy cows. Journal of Dairy Research, 70(1): 1-7, 2003.

Braun, U.; Schefer, U.; Gerber, D. Ultrasonography of the urinary tract of female sheep. American Journal of Veterinary Research, 53(10): 1734-1739, 1992.

Braun, U.; Steininger, K. Ultrasonographic characterization of the liver, caudal vena cava, portal vein, and gallbladder in goats. American Journal of Veterinary Research, 72(2): 219225, 2011.

Braun, U.; Jacquat, D.; Steininger, K. Ultrasonographic examination of the abdomen of the goat. II. Liver, spleen, urinary tract and greater omentum. Schweizer Archiv für Tierheilkund, 155(3): 185-195, 2013.

Braun, U.; Steininger, K. Ultrasonographic examination of the spleen in 30 goats. Schweizer Archiv für Tierheilkund, 152(10): 477-481, 2010.

Dyce, K.M.; Sack, W.O.; Wensing, C.J.C. Tratado de anatomia veterinária. $4^{\mathrm{a}}$ ed. Rio de Janeiro: Elsevier, 2010. 834p.

El-Kammar, M.A.; Alsafy, M.A.M.; Mohammed, M.I.; El-Neweshy, M.S. Ureteric obstruction and hydronephrosis in Baladi goats: topographical anatomy, ultrasonographical, hematological and pathological findings. Small Ruminant Research, 104(1-3): 169-178, 2012.

Fasulkov, I.R.; Georgiev, P.I.; Antonov, A.L.; Atanasov, A.S. B-mode ultrasonography of mammary glands in goats during the lactation period. Bulgarian Journal of Veterinary Medicine, 13(4): 245-251, 2010.

IBGE. Instituto Brasileiro de Geografia e Estatística. 2017. Disponível em: <https://sidra.ibge.gov.br/tabela/3939\#resultad o>. Acesso em: 24 fev. 2019.

Imran, S.; Tyagi, S.P.; Kumar, A.; Kumar, A.; Sharma, S. Comparative ultrasonographic imaging of spleen and liver in healthy crossbred cows. International Scholarly Research Network, 2011: 1-7, 2011.

Iswar, A.K. Pregnancy diagnosis in sheep and goats: a review. Small Ruminant Research, 17(1): 37-44, 1995.

Kandeel, A.E.; Omar, M.S.A.; Mekkawy, N.H.M.; El-Seddawy, F.D.; Gomaa, M. Anatomical and ultrasonographic study of the stomach and liver in sheep and goats. Iraqi Journal of Veterinary Science, 23(2): 181-191, 2009.

King, A.M. Development, advances and applications of diagnostic ultrasound in animals. The Veterinary Journal, 171(3): 408420, 2006.

Kofler, J.; Hittmair, K. Diagnostic ultrasonography in animals. Continuation of the clinical examination. Veterinary Journal, 171(3): 393395, 2006.

König, H.E.; Liebich, H.G. Anatomia dos animais domésticos. $4^{\mathrm{a}}$ ed. Porto Alegre: Artmed, 2011. $788 \mathrm{p}$.

Lindahl, I.L. Detection of pregnancy in sheep by means of ultrasound. Nature, 212: 642-643, 1966.

Maxson, A.D.; Wachira, T.M.; Zeyhle, E.E.; Fine, A.; Mwangi, T.W.; Smith, G. The use of ultrasound to study the prevalence of hydatid cysts in the right lung and liver of sheep and goats in Turkana, Kenya. International Journal for Parasitology, 26(11): 1335-1338, 1996.

Medan, M.S.; El-Aty, A.A.M. Advances in ultrasonography and its applications in domestic ruminants and other farm animals reproduction. Journal of Advanced Research, 1(2): 123-128, 2010.

Melo, C.H.S.; Sousa, F.C.; Batista, R.I.T.P.; Sanchez, D.J.D.; Sousa-Fabjan, J.M.G.; Freitas, V.J.F.; Teixeira, D.Í.A. Comparative analysis of laparoscopic and ultrasound-guided biopsy methods for gene expression analysis in transgenic goats. Genetics and Molecular Research, 14(3): 8672-8684, 2015.

Melo, C.H.S.; Sousa, F.C.; Teles Filho, A.C.A.; Moura, R.R.; Albuquerque, E.S.; Pereira, A. F.; Melo, L.M.; Freitas, V.J.F.; Teixeira, D.Í.A. Mensurações ultrassonográficas da cisterna da glândula mamária de caprino transgênico. Arquivo Brasileiro de Medicina Veterinária e Zootecnia, 64(2): 491-494, 2012.

Néspoli, P.B.; Gheller, V.A.; Mahecha, G.A.B.; Araújo, G.D.K.; Júnior, G.L.M.; Bordin, A.I. Aspectos morfológicos da ultra-sonografia hepática de ovinos. Pesquisa Veterinária Brasileira, 29(4): 333-338, 2009.

Newman, S.J.; Leichner, T.; Crisman, M.; Ramos, J. Congenital cystic disease of the liver and kidney in a pygmy goat. Journal of Veterinary Diagnostic Investigation, 12(4): 374-378, 2000. 
Nyland, T.G.; Mattoon, J.S. Veterinary diagnostic ultrasound. Philadelphia: Saunders, 1995. 357p.

Salama, A.A.K.; Caja, G.; Such, X.; Peris, S.; Sorensen, A.; Knight, C.H. Changes in cisternal udder compartment induced by milking interval in dairy goats milked once or twice daily. Journal of Dairy Science, 87(5): 1181-1187, 2004.

Simões, J. Os princípios físicos, a imagem e os artefatos na ecografia em modo B. 2008. Disponível em: <http://www.veterinaria.com.pt $>$. Acesso em: 11 mar. 2014.

Soroori, S.; Raoofi, A.; Vajhi, A.R.; Nezami, S.G. Ultrasonographic examination of the goat liver. Turkish Journal of Veterinary and Animal Sciences, 32(5): 385-388, 2008.

Sousa, F.C.; Melo, C.H.S.; Pereira, R.I.T.B.; Sanchez, D.J.D.; Souza-Fabjan, J.M.G.; Pereira, A.F.; Melo, L.M.; Freitas, V.J.F.; Teixeira, D.Í.A. Ultrasonographic findings of the mammary gland, liver, gallbladder, spleen, and kidneys in transgenic goats for hG-CSF during induced lactation. Semina: Ciências Agrárias, 37(6): 4109-4118, 2016.

Sousa, F.C.; Melo, C.H.S.; Teles Filho, A.C.A.; Avelar, S.R.G.; Moura, A.A.A.; Martins,
J.A.M.; Freitas, V.J.F.; Teixeira, D.Í.A. Ovarian follicular response to different hormonal stimulation treatments in Canindé goats. Animal Reproduction Science, 125(14): 8893, 2011.

Streeter, R.N.; Step, D.L. Diagnostic ultrasonography in ruminants. Veterinary Clinics of North America: Food Animal Practice, 23(3): 541-574, 2007.

Teixeira, D.Í.A.; Lopes Júnior, E.S.; Sousa, F.C.; Pinheiro, E.S.P.; Serova, I.A.; Andreeva, L.E.; Freitas, V.J.F. The use of real-time ultrasonography to select embryo donors participating in a transgenesis programme. Small Ruminant Research, 76(3): 215-219, 2008.

Teixeira, D.Í.A.; Pinheiro, E.S.P.; Sousa, F.C.; Pereira, A.F.; Freitas, V.J.F.; Rondina, D. Relação entre idade gestacional e parâmetros do concepto, mensurados por ultrassonografia em cabras AngloNubiana criadas no nordeste brasileiro. Ciência Animal, 19(1): 63-70, 2009.

Yamaga, Y.; Too, K. Diagnostic ultrasound imaging in domestic animals: Fundamental studies on abdominal organs and fetuses. Japanese Journal of Veterinary Science, 46(2): 203-212, 1984. 